\title{
Calculation of opacities and emisivities for carbon plasmas under NLTE and LTE conditions
}

\author{
J.M. Gil ${ }^{1, *}$, R. Rodríguez ${ }^{1}$, R. Florido ${ }^{1}$, J.G. Rubiano ${ }^{1}$, P. Sauvan², P. Martel ${ }^{1}$ \\ and $\mathrm{E}$. Mínguez ${ }^{3}$ \\ ${ }^{1}$ Departamento de Física de la Universidad de Las Palmas de Gran Canaria, \\ Campus Universitario de Tafira, 35017 Las Palmas de Gran Canaria, Spain \\ ${ }^{2}$ Departamento de Ingeniería Energética, Universidad Nacional de Educación a Distancia, \\ c/Juan del Roscal 12, 28004 Madrid, Spain \\ ${ }^{3}$ Instituto de Fusión Nuclear-DENIM, Universidad Politécnica de Madrid, c/José Gutiérrez \\ Abascal 2, 28006 Madrid, Spain
}

\begin{abstract}
We calculate different optical properties for carbon plasma in a wide range of temperatures and densities by using ATOM3R-OP code which has been recently developed. We have calculated average ionizations, level populations, opacities and emissivities and we focus our study on the identification with our code of Coronal Equilibrium, Non Local Thermodynamic Equilibrium and Local Thermodynamic Equilibrium regions for this kind of plasma. Moreover, we analyse the differences in the optical properties when they are calculated in Non Local Thermodynamic Equilibrium and Local Thermodynamic Equilibrium.
\end{abstract}

\section{INTRODUCTION}

ATOM3R-OP is a code recently developed to calculate optical properties for a wide range of temperatures and electron number densities. In this code we have implemented the rate equations, the Saha equation (LTE) and Coronal Equilibrium model (CE), and in a previous work [1] it has been shown that the ionic populations obtained by solving rate equations are in good agreement with those prodicted by LTE and $\mathrm{CE}$ at high and low electron density respectively.

In this work we make an study of optically thin hot carbon plasma for a wide range of temperatures and densities. First of all, we focus our study on the identification of the CE, NLTE and LTE regions for this kind of plasma by comparison of the average ionization and level populations. Secondly, we calculate the opacities and emissivities and we show the differences observed when the ionic populations are calculated using LTE, CE and rate equations. The atomic data used in this work are obtained from the FAC code [2] which provides relativistic energies, bound-bound oscillator strengths and bound-free cross sections.

\section{DESCRIPTION OF THE CALCULATIONS}

\subsection{ATOM3R-OP CODE}

ATOM3R-OP is a code to calculate optical properties for a wide range of temperatures and densities. This code obtains ionic populations by solving the steady state rate equations for optically thin and thick plasmas. For optically thick plasmas we consider homogeneus plasmas in planar, cylindrical and spherical geometries and the escape factor formalism is used. The opacities and emissivities are obtained

*Address correspondences: Juan Miguel Gil de la Fe, Departamento de Física, Universidad de Las Palmas de Gran Canaria, Campus de Tafira, Las Palmas de Gran Canaria 35017, Spain, e-mail: jmgil@dfis.ulpgc.es 
using Voigt profile for all spectral lines and assuming complete redistribution of the photons. The atomic data necesary to calculate the quantities mentioned above are obtained from different atomic models, i.e. selfconsistent or analytical models and from atomic databases. A large number of ionic configurations can be considered using sparse matrix techniques to store the matrix elements and iterative methods to solve the rate equations.

Table 1. Atomic Data.

\begin{tabular}{|c|c|c|c|c|}
\hline \hline & $\begin{array}{c}\text { Ionization } \\
\text { Potential (eV) }\end{array}$ & $\begin{array}{c}\text { Levels } \\
\text { Number }\end{array}$ & $\begin{array}{c}\text { B-B Transitions } \\
\text { Number }\end{array}$ & $\begin{array}{c}\text { B-F Transitions } \\
\text { Number }\end{array}$ \\
\hline CI & 11.2603 & 1285 & 34870 & 277 \\
\hline CII & 24.3833 & 104 & 744 & 2523 \\
\hline CIII & 47.8879 & 486 & 7720 & 4923 \\
\hline CIV & 64.4939 & 224 & 2565 & 3566 \\
\hline CV & 392.0872 & 449 & 6806 & 10087 \\
\hline CVI & 489.9933 & 225 & 2621 & 169 \\
\hline
\end{tabular}

\subsection{CARBON ATOMIC DATA}

The atomic data used in this work are obtained from FAC code which provides relativistic energies, bound-bound oscillator strengths and bound-free cross sections with a convenient specification of mixing schemes and it also includes configuration mixing. Some details of the atomic data are shown in the table 1. Finally, Krammer's formula is used for the free-free cross section and Voigt profile is used for all the spectral lines.

\section{RESULTS AND CONCLUSIONS}

We have determinated the average ionization of optically thin carbon plasmas in a range of temperatures and electron number densities given by $\left(10-200 \mathrm{eV}, 10^{14}-10^{24} \mathrm{~cm}^{-3}\right)$. In Figure 1 we show a map which allows to identify the density and temperature regions where our model predicts CE, LTE or NLTE. To establish these regions we have proceeded as it follows. If the average ionization calculated from CE/LTE

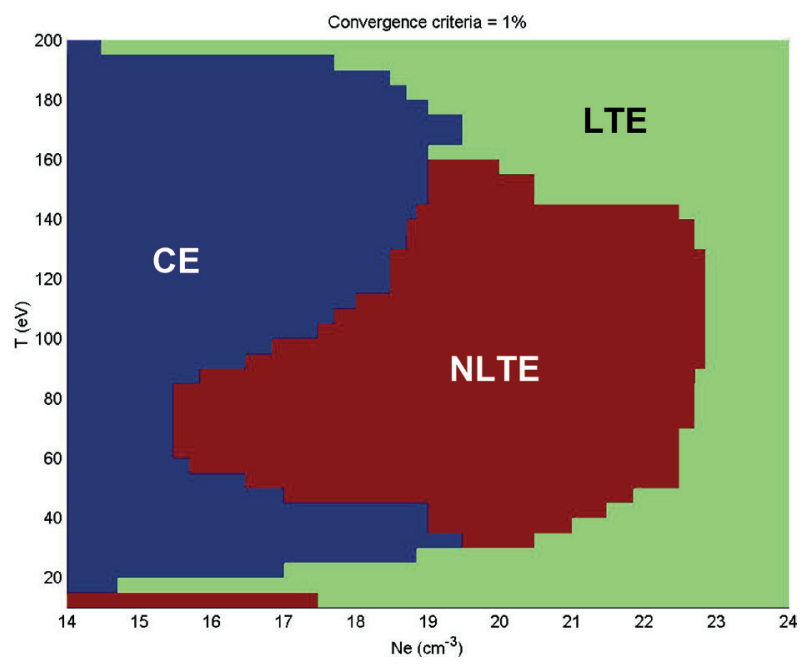

Figure 1. Map of CE, LTE and NLTE regimes determined from our level populations module. 

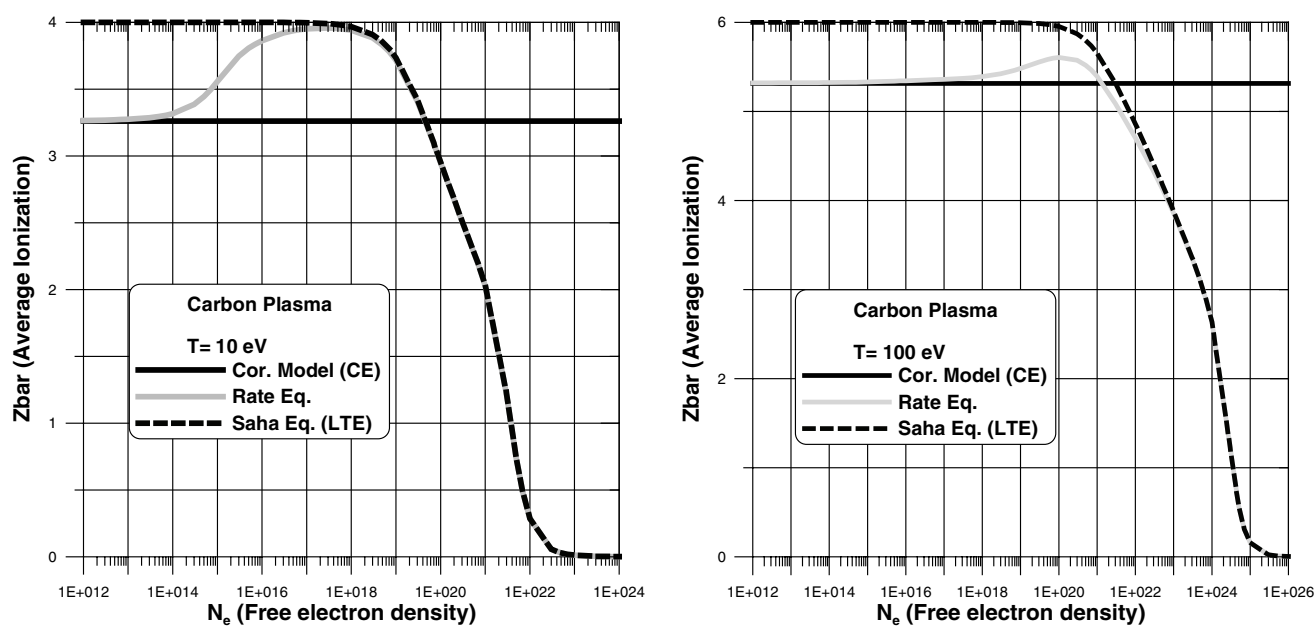

Figure 2. Average ionization vs free electron density calculated from steady state rate equation, Saha equations and Coronal Model Equations.
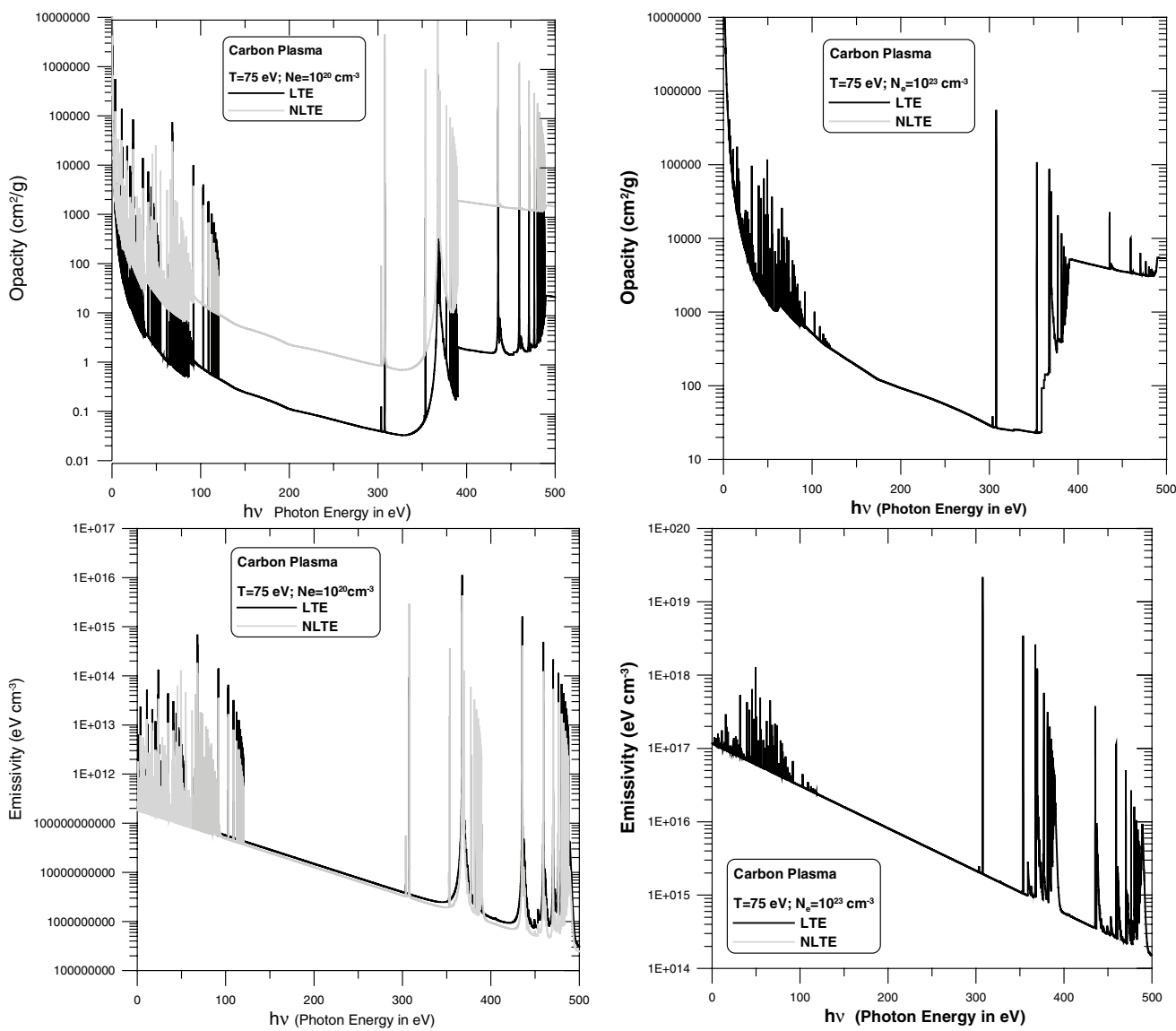

Figure 3. Opacities and emissivities for carbon plasma calculated in LTE and NLTE. 
is greater than $1 \%$ respect to that one obtained from rate equations then we consider NLTE regime. Else, we assume that CE or LTE has been achieved. Detailed information about this map is shown in Figure 2.

In Figure 3 we show the multifrequency opacities and emissivities asociated to two significant points (temperature and density) of the map given by Figure 1, one at NLTE $\left(75 \mathrm{eV}\right.$ and $\left.10^{20} \mathrm{~cm}^{-3}\right)$ and other at LTE $\left(75 \mathrm{eV}\right.$ and $\left.10^{23} \mathrm{~cm}^{-3}\right)$.

\section{Acknowledgments}

This work has been supported by the Project of the Spanish "Ministerio de Educación y Ciencia" with reference ENE2004-08184-C03-01/FTN, and by the program "Keep in Touch" of the "European Union".

\section{References}

[1] R. Florido, J.M. Gil, R. Rodríguez, J.G. Rubiano, P. Martel, E. Mínguez. Proceedings of the XXVIII European Conference on Laser Interaction with Matter, 389-413 (2004).

[2] Gu M.F., Astrophys. J. 582, 1241 (2003). 